\title{
Fatal anaphylaxis following multiple red fire ant strings
}

\author{
Radheshyam Purkait ${ }^{1}$, Biswajit Das ${ }^{1}$, Ashis Saha ${ }^{1}$, Sreyasi Bhattacharya ${ }^{1}$, \\ Monoranjan Mondal ${ }^{2}$, Birendranath Roy ${ }^{1}$ \\ (Department of Pediatric Medicine ${ }^{l}$ and Forensic Medicine ${ }^{2}$, NilRatan Sircar Medical College and Hospital, \\ India)
}

\begin{abstract}
Red Fire Ants Are Present In Almost All Countries And In All Places. Because Of Their Resistance To Natural And Chemical Control, Fire Ants Can Overwhelm Their Environment And Destroy Land And Animals. Fire Ants Can Also Cause A Variety Of Health Problems In Humans, Ranging From Simple Stings To Severe Life Threatening Anaphylaxis. Most Of The Deaths Following Red Fire Ant Strings Are Reported From South-Eastern United States And Australia. We Report A Three-Year-Old Child, Known Allergic To Ant-Venom, Who Died From Severe Anaphylactic Reactions Following Multiple Red Fire Ant Strings (Solenopsis Geminata). This Type Of Presentation Following Red Fire Ant Strings Is Probably First Time Reported From India. Beside Clinical Diagnosis, Significance Of Laboratory Investigations Including Postmortem Examinations In Fatal Anaphylaxis Is Also Discussed Here.
\end{abstract}

Keywords: Fatal Anaphylaxis; Red Fire Ant; Ant-Venom Allergy

\section{INTRODUCTION}

Ant-venom allergy that varies from minor local reaction to severe systemic reaction is a rare but known clinical problem and cases are reported from different part of the world including India. [1,2] Fatal anaphylaxis to ant-venom is one of the rarest incidences and most of the deaths are reported from south-eastern United States and Australia [3-5]. We present a three-year-old child who died of severe life threatening anaphylaxis following multiple red fire ant strings. At autopsy, multiple skin lesions characteristic of those produced by fire ant strings was present on her left arm. To the best of our knowledge such a case is almost never published from India.

\section{HEADING $S$}

1. Case History: A three-year-old male child was suddenly started crying while playing in the kitchen garden. His parents noticed that he was stung by eight-ten red fire ants on the left upper limb. He started itching and within few minutes he developed rashes, angioedema, breathlessness and wheezing. Immediately he was taken to a primary health center where the initial treatment was given. He was then referred to our hospital after developing anaphylactic shock. He suffered from similar attack in the past but developed mild local reaction and recovered after treatment with oral antihistaminic and steroid.

2. Examination: On admission child was obtunded with Glasgow coma score of $3 / 15$ having no apparent neurological deficit. On examination he had cold and clammy extremities with peripheral cyanosis, absent peripheral pulses and blood pressure was unrecordable with non-invasive blood pressure machine. His respiration was very poor and showed oxygen saturation of $60 \%$ in room air.

3. Investigations: A complete blood count on admission showed eosinophilia (18\%) with an absolute eosinophil count of 1548/ $\mu$. Total immunoglobulin (IgA, G and M) and complement levels were normal. Total serum IgE was $1846 \mathrm{IU} / \mathrm{ml}$ (reference range: $0-230 \mathrm{IU} / \mathrm{mL}$ ); however, venom specific IgE levels and tryptase were not was not done as the facility for the same was unavailable. Other investigations like liver and renal function tests, serum electrolytes and urine examination were within normal limits.

4. Treatment: Immediately he was intubated and started mechanical ventilation with $100 \%$ oxygen. Besides oxygen, adrenaline, diphenhydramine, $0.9 \%$ saline, dopamine and hydrocortisone were given intravenously. A repeat bolus dose of Inj adrenaline was given as the peripheral pulses did not return after initial resuscitation. But despite the entire supportive management child died within one hour after admission.

5. Follow-up: A police case was registered, and the body of the child was sent for a medico-legal autopsy to find out the exact cause of death. Autopsy examination revealed the deceased child appeared well nourished with multiple maculo-papular erythematous skin rashes over left upper limb consistent with ant string [Figure 1]. The rest of the external and internal examination was essentially normal, except for petechial hemorrhages of many of the viscera and mild laryngeal edema. 


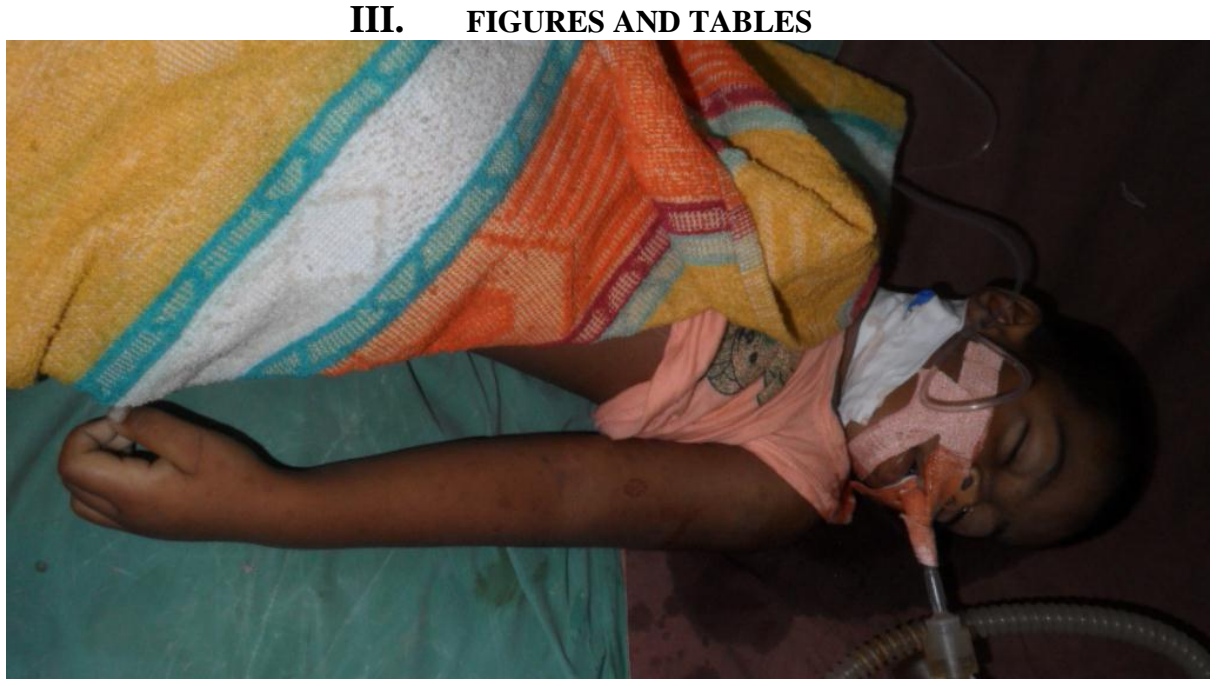

Fig.1: Showing multiple maculo-papular erythematous skin rashes over left upper limb characteristic of red fire ant string

\section{CONCLUSION}

Fire ant are stringing insects that belongs to the order hymenoptera, members of genus Solenopsis namely S. invicta, S. richteri and S. geminata. The red (Solenopsis invicta) and black (Solenopsis richteri) imported fire ants are the source of a potentially lethal environmental hazard in United States whereas Solenopsis geminata become the major health concern worldwide including tropical countries. Though many species of ants exist in India but the present report was due to red fire ant (Solenopsis geminate (fabricius)) string as verified by entomologist after collecting them from the site of accident. Red fire ant is present mostly in the kitchen gardens and farm land all over India. Because of their resistance to natural and chemical control, fire ants can overwhelm their environment, causing numerous stinging accidents $[1,6]$.

Alkaloid constitutes the major component (95\%) of red fire ant-venom with a small aqueous fraction that contains soluble proteins. About ninety-nine percent of the venom alkaloid is made up of 2,6,di-substituted piperidines which have hemolytic, antibacterial, insecticidal, and cytotoxic properties. Venom alkaloid carries less importance antigenecally as they do not induce any IgE mediated allergic responses (Type-1). Only the proteins component in fire ant-venom which make up only about $0.1 \%$ of the venom by the weight can induce Type 1 hypersensitivity in persons allergic to fire ant stings [2].

Stinging induced anaphylaxis is more common among male children and they occur most frequently in August through October. Reactions to fire ant sting range from localized itching and swelling with pustule formation to severe, life-threatening anaphylaxis. Severe reactions are usually more experienced in persons sensitized by a previous string [7]. Our index case had ant-sting allergy. Anaphylaxis may occur within 30 to 60 minutes after the sting and may be manifested by flushing, generalized hives, swelling of the face, eyes or throat, difficulty breathing, or loss of consciousness. Other serious adverse reactions like laryngospasm, seizures and rhabdomyolysis with renal failure have also been reported in the previous literature [8].

No optimal and readily available laboratory test can confirm the clinical diagnosis of an anaphylactic episode. However, in some patients the clinical diagnosis of anaphylaxis can be confirmed by means of a blood test; for example, an increased serum total tryptase level. Positive skin test results with ant-venom extract or increased serum venom-specific IgE levels verify sensitization but do not confirm the diagnosis of anaphylaxis because asymptomatic sensitization is common in the general population. Even on postmortem examination, the findings are non-specific in most of the cases of fatal anaphylaxis and do not authenticate the diagnosis $[9,10]$.

Anaphylactic reactions should be managed as a medical emergency. Adrenalin (epinephrine) is the first drug of choice in the treatment of anaphylaxis. Further treatment may include antihistamines and corticosteroids. Persons suffering fire ant sting anaphylaxis should be referred to an allergist for skin testing and immunotherapy. All insect sting-allergic patients should carry an emergency kit containing epinephrine (e.g., EpiPen or AnaKit), a written, personalized anaphylaxis emergency action plan; and up-to-date medical identification [9].

\section{REFERENCES}

[1]. Havaldar PV, Patil SS, Phadnis C. Anaphylaxis due to red fire ant bite. Indian Pediatr. 2012;49(3):237-8.

[2]. Nandhakumar V. Angioedema following ingestion of fried flying red fire ants. Indian Pediatr. 2013;50(4):423-4.

[3]. Prahlow JA, Barnard JJ. Fatal anaphylaxis due to fire ant stings. Am J Forensic Med Pathol. 1998;19(2):137-42. 
[4]. More DR, Kohlmeier RE, Hoffman DR. Fatal anaphylaxis to indoor native fire ant stings in an infant. Am J Forensic Med Pathol. 2008;29(1):62-3.

[5]. Brown SG, Wu QX, Kelsall GR, Heddle RJ, Baldo BA. Fatal anaphylaxis following jack jumper ant sting in southern Tasmania. Med J Aust. 2001;175(11-12):644-7.

[6]. McGlynn TP. The worldwide transfer of ants: geographical distribution and ecological invasions. J Biogeography. 1999;26(3):53548.

[7]. Harduar-Morano L, Simon MR, Watkins S, Blackmore C. A population-based epidemiologic study of emergency department visits for anaphylaxis in Florida. J Allergy Clin Immunol. 2011;128(3):594-600.

[8]. Koya S, Crenshaw D, Agarwal A. Rhabdomyolysis and acute renal failure after fire ant bites. J Gen Intern Med. 2007;22(1):145-7.

[9]. Simons FE. Anaphylaxis. J Allergy Clin Immunol. 2010;125 (2 Suppl 2):S161-81.

[10]. Pumphrey RS, Roberts IS. Postmortem findings after fatal anaphylactic reactions. J Clin Pathol. 2000;53(4):273-6. 